\title{
SUPERCRITICAL TWO-FLUID INTERACTIONS WITH SURFACE TENSION AND GRAVITY
}

\author{
JEAN-MARC VANDEN-BROECK AND FRANK T. SMITH
}

\begin{abstract}
Gravity and surface-tension effects are examined for inviscidinviscid interactions between two fluids close to a wall. The ratios of density and viscosity of the two fluids are taken to be small. A nonlinear integro-differential equation is found to govern the near-wall flow velocity, interface shape and pressure; analysis, computation and comparisons are then applied. Travelling-state solutions are of particular interest.
\end{abstract}

$\S 1$. Introduction. Interaction between two fluids near a fixed solid surface is studied here, with gravity and surface tension acting as substantial influences on the assumed planar motion. The two fluids are immiscible and incompressible and have small density and viscosity ratios. The heavier, more viscous body of fluid is approaching the solid surface, and the other fluid is lying as a thin layer in between. In the so-called supercritical range where, for both fluids, inviscid forces dominate over viscous ones, a trio of pressure-shape-velocity relations is found which leads to a nonlinear integro-differential equation for the unknown interface shape. As regards motivation and application, the possible implications for air-water interaction close to a wall and the associated impact problems are of much interest. One scenario is that of a droplet of water (fluid 1, say) rapidly approaching a wall, with air (fluid 2) between the droplet and the wall, and the subsequent impact onto the wall; the two fluids mentioned here for generality have small density and viscosity ratios. Air-water interactions are examined experimentally and numerically in $[3,7,12-14]$, with numerous other aspects being investigated in $[\mathbf{5}, \mathbf{6}, \mathbf{8 - 1 1}, \mathbf{1 5}, \mathbf{1 9}, \mathbf{2 1}, \mathbf{2 2}, \mathbf{2 4}]$. The background for the water-air-wall setting, or more generally the fluid 1-fluid 2wall setting, includes a recent upsurge of industrial interest-for instance, in food manufacture, spray coating and aeronautics, the last of which concerns aircraft or rotorcraft icing $[2,16,17,20]$.

Above a critical Reynolds number identified in [19], the air acts as if inviscid, whereas below this critical value the air acts as if it were a lubricating fluid. The surface-tension effect stands out because it suggests that the general two-fluid interaction leads to a nonlinear developing steady state or travelling wave at large scaled time, if such surface tension is not negligible. Paper [15] addresses interactions for super-critical Reynolds numbers, where the inviscid-inviscid interaction between the two fluids tends to be very unstable to short length 
disturbances unless surface tension acts to stabilize these interactions. Postimpact behaviour of two-fluid interaction is considered in [20] and [15], with the latter incorporating the influence of surface tension locally close to a contact point and finding a new form of local interface behaviour, while travelling wave solutions were described recently in [23]. Clearly, surface tension has significant influence in several areas of concern; our particular focus is on the pre-impact stage, where a thin layer of fluid 2 first enters play as a droplet of fluid 1 approaches a fixed solid wall, in the super-critical regime of Reynolds numbers.

Gravity effects are of interest in their own right. They can also be significant in terms of distinguishing between two-fluid behaviour on top of a solid surface and underneath a solid surface, as well as the different responses to gravity force at different angles of inclination of the surface. The current study concentrates on horizontal layers. Further, in planetary physics, strong gravitational effects can in fact be dominant forces in the fluid responses near a planet surface.

Section 2 describes the basis of the argument concerning small density and viscosity ratios which, in the super-critical range and with gravity and surface tension forces being effective, leads to the two-fluid interaction depending on inviscid forces in fluid 2 coupled with potential flow dynamics in fluid 1. A nonlinear integro-differential system is obtained for the evolution of the interface and induced pressure. This is described in $\$ 2$ along with details about the critical Reynolds number. Travelling-wave and other related forms of solution are presented in $\S 3$, followed by further comments in $\$ 4$ on the present theoretical investigation.

$\S 2$. Small-ratio argument. First, in the non-dimensionalization, the velocity $\mathbf{u}=(u, v)$, the corresponding Cartesian coordinates $(x, y)$, the pressure $p$ and the time $t$ are based on the droplet approach speed $V$, a representative length scale $L, \rho_{1} V^{2}$ and $L / V$, respectively. The length $L$ is a global quantity such as the droplet diameter if the droplet is of circular shape, while $\rho_{1}$ and $\rho_{2}$ are the densities of the fluid 1 and fluid 2 , respectively, and $\mu_{1}\left(=\rho_{1} \nu_{1}\right)$ and $\mu_{2}\left(=\rho_{2} \nu_{2}\right)$ are their respective viscosities. For convenience, the coordinates used are centred in the impact area and the pressure is measured relative to the general atmospheric value for fluid 2. Also, $\operatorname{Re}_{1}=V L / v_{1}$ is the droplet Reynolds number, $\mathrm{We}_{1}$ is the corresponding Weber number, and $\mathrm{Fr}_{1}$ is the Froude number. Here $\nabla$ denotes the operator $\left(\partial_{x}, \partial_{y}\right)$, and three-dimensionality and compressibility are neglected. The continuity equation then reads, for either fluid,

$$
\nabla \cdot \mathbf{u}=0
$$

while the Navier-Stokes equations in non-dimensional variables take the form

$$
\left(\partial_{t}+\mathbf{u} \cdot \nabla\right) \mathbf{u}=-\nabla p+\operatorname{Re}_{1}^{-1} \nabla^{2} \mathbf{u}
$$

in fluid 1 and

$$
\left(\partial_{t}+\mathbf{u} \cdot \nabla\right) \mathbf{u}=-\left(\rho_{1} / \rho_{2}\right) \nabla p+\operatorname{Re}_{1}^{-1}\left(\nu_{2} / \nu_{1}\right) \nabla^{2} \mathbf{u}
$$

in fluid 2. 


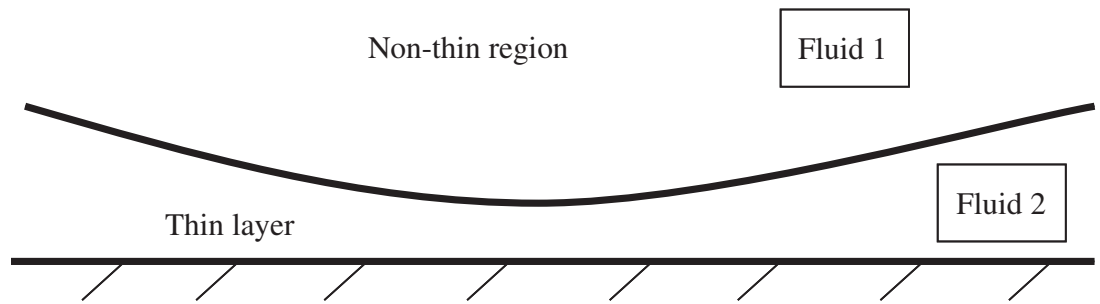

Figure 1: Sketch of the flow.

Second, the density and viscosity ratios of the two fluids, $\rho_{2} / \rho_{1}$ and $\mu_{2} / \mu_{1}$, are supposed to be small and comparable with each other so that, typically, the ratio $v_{2} / \nu_{1}$ can be considered to be of order unity. The flow solution then expands according to the form

$$
\begin{array}{cc}
(u, v, p)=\left(u_{1}, v_{1}, h^{-1} p_{1}\right)+\cdots & \text { in fluid } 1 \\
(u, v, p)=\left(h^{-1} u_{2}, v_{2}, h^{-1} p_{2}\right)+\cdots & \text { in fluid } 2
\end{array}
$$

with $h(\ll 1)$ as the main expansion parameter (see Figure 1).

The expansions follow from an order-of-magnitude argument, given that: the length scales in fluid 1 are short near impact, such that $(x, y)=(X, Y) a / L$ where the characteristic local length $a$ is much smaller than $L$; the layer of fluid 2 that lies astride the $x$-axis has length scales which are $(x, y)=\left(X, h y_{2}\right) a / L$; for a smooth incident droplet shape, $a / L \approx h$; the typical time scale $t=h^{2} T$ is also short, with $T \approx 1$, because of the $O(1)$ approach speed and the $h^{2}$ thickness of the fluid-2 layer. The aspect ratio $h$ of the fluid-2 layer is assumed to be small, in the present setting of an impact with rapid local interaction involving the relatively thin layer of fluid 2 between fluid 1 and a solid surface or possibly between two bodies of fluid 1, depending on whether the droplet impacts on a solid or on another body of fluid 1 .

Third, the resulting equations of motion in fluid 1 are those of unsteady potential flow:

$$
\frac{\partial u_{1}}{\partial X}+\frac{\partial v_{1}}{\partial X}=0, \quad \frac{\partial u_{1}}{\partial T}=-\frac{\partial p_{1}}{\partial X}, \quad \frac{\partial v_{1}}{\partial T}=-\frac{\partial p_{1}}{\partial Y}
$$

to leading order. In fluid 2, the controlling equations of an unsteady inviscid thin layer hold:

$$
\begin{gathered}
\frac{\partial u_{2}}{\partial X}+\frac{\partial v_{2}}{\partial y_{2}}=0, \quad \frac{\partial u_{2}}{\partial T}+u_{2} \frac{\partial u_{2}}{\partial X}+v_{2} \frac{\partial u_{2}}{\partial y_{2}}=-H\left(\frac{\partial p_{2}}{\partial X}-D \frac{\partial F}{\partial X}\right), \\
0=-\frac{\partial p_{2}}{\partial y_{2}} .
\end{gathered}
$$

Here, the constant $D=g a^{3} /(L V)^{2}$ is taken to be of $O(1)$, corresponding to the Froude number $\operatorname{Fr}_{1}=V /(g L)^{1 / 2}$ being small of order $(a / L)^{3 / 2}$, where $g$ denotes gravity. The constant $H=h \rho_{1} / \rho_{2}$ is also assumed to be of order 
unity, so that the small quantities $h$ and $\rho_{2} / \rho_{1}$ can be taken as comparable. Matching at the unknown interface defined as $y_{2}=F(X, T)$ requires, among other conditions, the difference between the fluid-1 pressure $p_{1}(X, Y=0+, T)$ and the fluid-2 pressure $p_{2}(X, T)$-which is independent of $y_{2}$ by the third equation in (7), is also unknown and will be denoted by $P(X, T)$ - to be due to a surface-tension effect (surface tension multiplied by the interfacial curvature, which is proportional to $\partial^{2} F / \partial X^{2}$ in the present context), an effect additional to the gravity effect that is proportional to $F$ in terms of pressure. Tangentialvelocity, kinematic and tangential-stress conditions also apply at that interface. The boundary conditions at large $Y$ in fluid 1 require no exponential growth.

The validity of (6)-(7) relies on several assumptions which may or may not be met in a specific real application. These are, mainly: $h$ is of the order of $\rho_{2} / \rho_{1}$, as anticipated earlier; the global Reynolds number $\operatorname{Re}_{1}$ is large; the local Reynolds number $\operatorname{Re}_{1} h$ is large compared with $\mu_{2} /\left(\mu_{1} h^{2}\right)$. It follows that the theory applies for values of the global Reynolds number $\operatorname{Re}_{1}$ that are large and lie above approximately $\left(\rho_{1}^{2} v_{2}\right) /\left(\rho_{2}^{2} v_{1}\right)$. This critical value of $\operatorname{Re}_{1}$ is greater than 10 million for the air-water combination mentioned in the introduction.

From (6), (7) and the interfacial and other conditions, the two-fluid interaction here is controlled by coupled equations for the unknown scaled interface shape $F(X, T)$, the pressure $P(X, T)$ and the velocity component $U(X, T)$, namely:

$$
\begin{gathered}
\pi F_{T T}=(\mathrm{PV}) \int_{-\infty}^{\infty}\left[P_{s}(s, T)+\sigma F_{s s s}(s, T)-D F_{s}(s, T)\right](X-s)^{-1} d s \\
U_{T}+U U_{X}=-H P_{X}, \quad F_{T}+(U F)_{X}=0 .
\end{gathered}
$$

These are subject to $u_{2}=U(X, T)$ being independent of $y_{2}$ and to the kinematic constraint which yields the second of the equations (9). In (8), "(PV)" stands for the principal value of the Cauchy-Hilbert integral, which runs from $-\infty$ to $\infty$. Equation (8) follows from the fluid-1 flow equations, while equations (9) are from the fluid-2 motion. The parameter $\sigma$ is the dimensional surface tension multiplied by $h^{2} /\left(\rho_{1} V^{2} a\right)$; it is of order $h / \mathrm{We}_{1}$ and is taken to be of $O(1)$. To be strict, therefore, a small Weber number is assumed in the theory, as well as a small Froude number; relatively large gravity forces substantially affect the fluid-1 dynamics here because of the density ratio. Inside fluid 2, the scaled pressure $P$ must tend to zero at large positive or negative $X$, in view of the general atmospheric pressure, whereas the pressure in fluid 1 tends to $2 \sigma$.

For zero $\sigma$ and $D$, solutions of $(8,9)$ are discussed in $[15]$ with the normalized condition

$$
F \approx X^{2}-T, \quad P \rightarrow 0 \text { as }|X| \rightarrow \infty
$$

for an incident locally parabolic shape of the interface when or where interaction is still weak, and in line with $v \rightarrow-1$ in the incident motion. The solutions show that as the interaction strengthens, it leads to a short-scale catastrophic instability and likely touchdown; see [19]. A limited number of solutions with non-zero $\sigma$ given in [15] suggest that touchdown does not occur within a finite time. These set the scene for the recent study of $\sigma$ effects in [23] as well as the following investigation of travelling states for non-zero $\sigma$ and $D$. 
Finally, we seek solutions of $(8,9)$ in travelling-wave form such that $F, P$ and $U$ depend only on $X-c T$ ( $=\xi$, say), rather than on $X$ and $T$ separately. Here $c$ is the constant scaled velocity of the solution. The system therefore reduces to

$$
\begin{gathered}
c^{2} \pi f_{\xi \xi}=(\mathrm{PV}) \int_{-\infty}^{\infty}\left[P_{s}(s)+\sigma F_{s s s}(s)-D F_{s}(s)\right](\xi-s)^{-1} d s, \\
-c U_{\xi}+U U_{\xi}=-H P_{\xi}, \quad-c F_{\xi}+(U F)_{\xi}=0
\end{gathered}
$$

for $F(\xi), P(\xi)$ and $U(\xi)$. Hence, the nonlinear integro-differential equation

$$
c^{2} \pi F^{\prime \prime}=(\mathrm{PV}) \int_{-\infty}^{\infty}\left[C_{1} \frac{F^{\prime}}{F^{3}}+\sigma F^{\prime \prime \prime}(s)-D F^{\prime}(s)\right](\xi-s)^{-1} d s
$$

is obtained for the unknown interfacial function $F(\xi)$, where prime denotes differentiation and the non-negative constant $C_{1}=H^{-1} c_{1}^{2}$ is a constant of integration arising in the relations that stem from (11) and (12).

\section{§3. Periodic solutions.}

3.1. Weakly nonlinear theory. We seek solutions of (13) by assuming expansions of the form

$$
F(\xi)=A+\epsilon F_{1}(\xi)+\epsilon^{2} F_{2}(\xi)+\cdots
$$

and

$$
c=c_{0}+\epsilon c_{1}+\epsilon^{2} c_{2}+\cdots .
$$

Here $A$ (which is positive and of order unity) and $0<\epsilon \ll 1$ are constants. Substituting (14) and (15) into (13), expanding in powers of $\epsilon$ and keeping terms up to order $\epsilon^{2}$ yields

$$
\begin{aligned}
& \epsilon c_{0}^{2} \pi F_{1}^{\prime \prime}+2 \epsilon^{2} c_{0} c_{1} \pi F_{1}^{\prime \prime}+\epsilon^{2} c_{0}^{2} \pi F_{2}^{\prime \prime} \\
& =(\mathrm{PV}) \int_{-\infty}^{\infty}\left[\frac{\epsilon C_{1} F_{1}^{\prime}}{A^{3}}+\epsilon^{2} C_{1}\left(\frac{F_{2}^{\prime}}{A^{3}}-\frac{3 F_{1} F_{1}^{\prime}}{A^{4}}\right)+\epsilon \sigma F_{1}^{\prime \prime \prime}\right. \\
& \left.+\epsilon^{2} \sigma F_{2}^{\prime \prime \prime}-\epsilon D F_{1}^{\prime}-\epsilon^{2} D F_{2}^{\prime}\right](\xi-s)^{-1} d s .
\end{aligned}
$$

Equating the coefficients of $\epsilon$ and $\epsilon^{2}$ in (16) gives the two equations

$$
\begin{aligned}
& c_{0}^{2} \pi F_{1}^{\prime \prime}=(\mathrm{PV}) \int_{-\infty}^{\infty}\left[\frac{C_{1} F_{1}^{\prime}}{A^{3}}+\sigma F_{1}^{\prime \prime \prime}-D F_{1}^{\prime}\right](\xi-s)^{-1} d s \\
& 2 c_{0} c_{1} \pi F_{1}^{\prime \prime}+c_{0}^{2} \pi F_{2}^{\prime \prime}=(\mathrm{PV}) \int_{-\infty}^{\infty}[ {\left[C_{1}\left(\frac{F_{2}^{\prime}}{A^{3}}-\frac{3 F_{1} F_{1}^{\prime}}{A^{4}}\right)+\sigma F_{2}^{\prime \prime \prime}-D F_{2}^{\prime}\right] } \\
& \times(\xi-s)^{-1} d s .
\end{aligned}
$$

We shall assume that the solutions are symmetric with respect to $\xi=0$. Since we are seeking periodic solutions, we then represent $F_{1}$ and $F_{2}$ by Fourier cosine 
series as follows:

$$
\begin{aligned}
& F_{1}=\sum_{n=1}^{\infty} a_{n} \cos n k \xi, \\
& F_{2}=\sum_{n=1}^{\infty} b_{n} \cos n k \xi,
\end{aligned}
$$

where $k=2 \pi / \lambda$ is the wavenumber and $\lambda$ is the wavelength.

We now define $\epsilon$ by the relation

$$
\epsilon=a k
$$

where

$$
a=\frac{2}{\lambda} \int_{0}^{\lambda} F(\xi) \cos k \xi d \xi
$$

is the first Fourier coefficient of $F(\xi)$. It then follows from (19) and (20) that

$$
a_{1}=\frac{1}{k}, \quad b_{1}=0 .
$$

We now substitute (19) into (17) and equate coefficients of $\cos n k \xi$. Using the identity

$$
\int_{-\infty}^{\infty} \sin n k s(\xi-s)^{-1} d s=-\pi \cos n k \xi
$$

we obtain

$$
a_{n}\left[-c_{0}^{2} n^{2} k^{2} \pi-\frac{C_{1} n k \pi}{A^{3}}+\pi \sigma n^{3} k^{3}+\pi D n k\right]=0, \quad n=1,2,3, \ldots
$$

Since $a_{1}=1 / k \neq 0$, relation (25) with $n=1$ implies that

$$
c_{0}^{2}=-\left(\frac{C_{1}}{A^{3}}-D\right) \frac{1}{k}+\sigma k .
$$

Similarly, relation (25) implies that

$$
a_{n}=0 \quad \text { for } n=2,3,4, \ldots
$$

provided that

$$
-c_{0}^{2} n^{2} k^{2}-\frac{C_{1} n k}{A^{3}}+\sigma n^{3} k^{3}+D n k \neq 0 \quad \text { for } n=2,3, \ldots
$$

If (28) is not satisfied for some values $n=m$, i.e. if

$$
-c_{0}^{2} m^{2} k^{2}-\frac{C_{1} m k}{A^{3}}+\sigma m^{3} k^{3}+D m k=0,
$$

then $a_{m}$ is arbitrary. 
We note that (28) and (29) can be written in the simpler forms

$$
\left(D-\frac{C_{1}}{A^{3}}\right) \frac{1}{\sigma k^{2}} \neq n \quad \text { for } n=2,3, \ldots
$$

and

$$
\left(D-\frac{C_{1}}{A^{3}}\right) \frac{1}{\sigma k^{2}}=m
$$

by using (26).

In summary, to leading order we have

$$
F_{1}(\xi)=a_{1} \cos k \xi
$$

when (28) (or (30)) is satisfied and

$$
F_{1}(\xi)=a_{1} \cos k \xi+a_{m} \cos m k \xi
$$

if (29) (or (31)) holds for some integer $m \geq 2$. As we shall see, the value of the arbitrary constant $a_{m}$ is determined by considering the problems at higher order in $\epsilon$.

3.1.1. Second-order solution when (28) holds. In this subsection we assume that (28) holds and calculate $F_{2}$. Upon substituting (20) and (32) into (18) and using (24), we obtain

$$
\begin{aligned}
& -2 c_{0} c_{1} a_{1} \pi k^{2} \cos k \xi-c_{0}^{2} \pi k^{2} \sum_{n=2}^{\infty} b_{n} n^{2} \cos n k \xi \\
& =(\mathrm{PV}) \int_{-\infty}^{\infty}\left[\frac{-C_{1}}{A^{3}} k \sum_{n=2}^{\infty} b_{n} n \sin n k s+\frac{3 C_{1}}{2 A^{4}} a_{1}^{2} k \sin 2 k s\right. \\
& \left.+\sigma k^{3} \sum_{n=2}^{\infty} b_{n} n^{3} \sin n k s+D k \sum_{n=2}^{\infty} b_{n} n \sin n k s\right](\xi-s)^{-1} d s .
\end{aligned}
$$

Equating the coefficients of $\cos k \xi, \cos 2 k \xi$ and $\cos n k \xi$ for $n=3,4, \ldots$ yields

$$
\begin{gathered}
c_{1}=0, \\
b_{2}=\frac{3 k C_{1} a_{1}^{2}}{2 A^{4}} \frac{1}{4 k^{2} c_{0}^{2}+2 C_{1} k / A^{3}-8 \sigma k^{3}-2 D k}, \\
b_{n}=0 \quad \text { for } n=3,2, \ldots
\end{gathered}
$$

Therefore (20) reduces to

$$
F_{2}(\xi)=b_{2} \cos 2 k \xi
$$

where $b_{2}$ is defined by (36). We note that the denominator of the second fraction in the right-hand side of (36) vanishes when (31) is satisfied for $m=2$. There is no contradiction since we have assumed that (28) holds. 
In conclusion, the solution up to second order is given by

$$
\begin{gathered}
c=c_{0}+O\left(\epsilon^{3}\right), \\
F=A+\epsilon F_{1}+\epsilon^{2} F_{2}+O\left(\epsilon^{3}\right),
\end{gathered}
$$

where $c_{0}, F_{1}$ and $F_{2}$ are defined by (26), (32) and (38), respectively.

3.1.2. Solutions when (28) does not hold. When (28) does not hold, i.e. when (29) (or, equivalently, (31)) is satisfied for some integer $m$, the firstorder solution is given by (33) instead of (32). In this section we show how to determine the arbitrary coefficient $a_{m}$. For simplicity of presentation we assume that $m=2$. Relation (31) then takes the form

$$
\left(D-\frac{C_{1}}{A^{3}}\right) \frac{1}{\sigma k^{2}}=2 .
$$

Solutions for $m>2$ will be computed numerically in $\$ 3.2$.

Substituting

$$
F_{1}(\xi)=a_{1} \cos k \xi+a_{2} \cos 2 k \xi
$$

and (20) into (18) gives, after some algebra,

$$
\begin{gathered}
-2 c_{0} c_{1} a_{1} \pi k^{2} \cos k \xi-8 c_{0} c_{1} a_{2} \pi k^{2} \cos 2 k \xi-c_{0}^{2} \pi k^{2} \sum_{n=2}^{\infty} b_{n} n^{2} \cos n k \xi \\
=\int_{-\infty}^{\infty}\left[\frac{-C_{1}}{A^{3}} k \sum_{n=2}^{\infty} b_{n} n \sin n k s+\frac{3 C_{1}}{2 A^{4}} a_{1}^{2} k \sin 2 k s+\frac{3 C_{1}}{2 A^{4}} a_{1} a_{2} k \sin k s\right. \\
+\frac{9 C_{1}}{2 A^{4}} a_{1} a_{2} k \sin 3 k s+\frac{3 C_{1}}{2 A^{4}} a_{2}^{2} k \sin 4 k s+\sigma k^{3} \sum_{n=2}^{\infty} b_{n} n^{3} \sin n k s \\
\left.+D k \sum_{n=2}^{\infty} b_{n} n \sin n k s\right](\xi-s)^{-1} d s .
\end{gathered}
$$

Upon equating coefficients of $\cos k \xi$ and $\cos 2 k \xi$ in (43), we get

$$
\begin{aligned}
& 2 c_{0} c_{1} k=\frac{3}{2 A^{4}} a_{2} C_{1}, \\
& -8 c_{0} c_{1} a_{2} \pi k^{2}+b_{2}\left(-4 c_{0}^{2} \pi k^{2}-\frac{2 C_{1}}{A^{3}} k \pi+8 \sigma k^{3} \pi+2 D k \pi\right) \\
& =-\frac{3 C_{1}}{2 A^{4}} a_{1}^{2} k \pi .
\end{aligned}
$$

Using (29), with $m=2$, equation (45) reduces to

$$
-8 c_{0} c_{1} a_{2} k^{2}=-\frac{3 C_{1}}{2 A^{4}} a_{1}^{2} k .
$$

Solving (44) with respect to $c_{1}$ and substituting into (46) gives

$$
a_{2}^{2}=\frac{a_{1}^{2}}{4}
$$




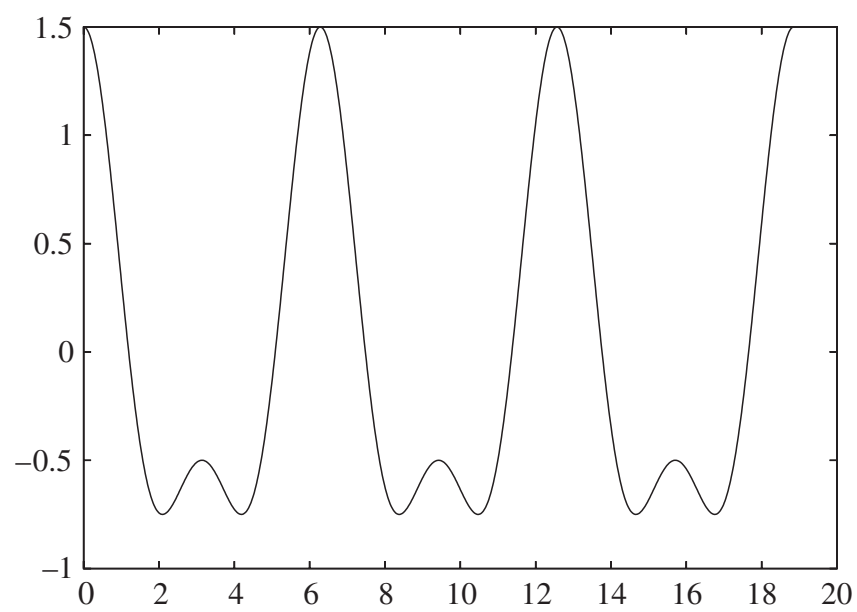

Figure 2: Values of $F(\xi)-A$ versus $\xi$ with $\epsilon a_{1}=1$ and $k=1$. This corresponds to the + sign in (50).

or

$$
a_{2}= \pm \frac{a_{1}}{2}
$$

Using (44) and (48), we obtain

$$
c_{1}= \pm \frac{3 C_{1} a_{1}}{8 A^{4} c_{0} k} .
$$

This concludes the determination of the solution up to order $\epsilon$. It is given by

$$
F(\xi)=A+\epsilon\left(a_{1} \cos k \xi \pm \frac{a_{1}}{2} \cos 2 k \xi\right)+O\left(\epsilon^{2}\right)
$$

and

$$
c=c_{0}+\epsilon c_{1}+O\left(\epsilon^{2}\right),
$$

where $a_{1}, c_{0}$ and $c_{1}$ are defined by (23), (26) and (49), respectively.

The profiles (50) are sketched in Figures 2 and 3.

We note that solutions similar to those presented in Figures 2 and 3 occur in the theory of water waves when both gravity and surface tension are taken into account. In that context they are referred to as Wilton ripples (see $[\mathbf{1 , 4 , 1 8 , 2 5 ] ) .}$

3.2. Nonlinear solutions. We seek nonlinear periodic solutions of (13) by representing $F(\xi)$ in terms of the Fourier series

$$
F(\xi)=A+\sum_{n=1}^{\infty} f_{n} \cos n k \xi .
$$

Here we have assumed that the wave is symmetric and that the origin $\xi=0$ is chosen so that $F(\xi)$ is even. There are then no $\sin n k \xi$ terms in the Fourier series (52). 


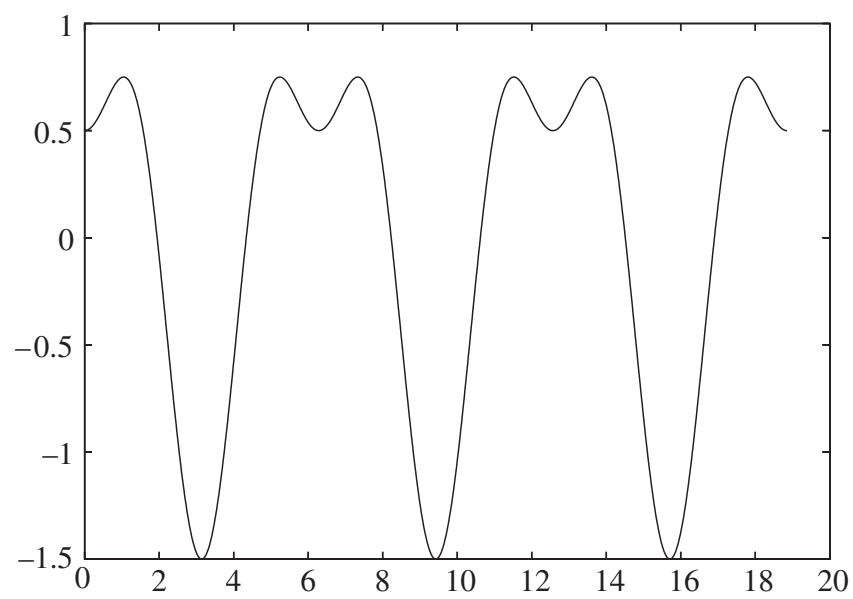

Figure 3: Values of $F(\xi)-A$ versus $\xi$ with $\epsilon a_{1}=1$ and $k=1$. This corresponds to the sign in (50).

The integral on the right-hand side of (13) is of the form

$$
\int_{-\infty}^{\infty} B(s)(\xi-s)^{-1} d s
$$

where $B(s)$ is a periodic function of period $\lambda=2 \pi / k$. Furthermore, $B(s)$ is odd, i.e. $B(-s)=-B(s)$. By summation of the contributions from all the periods, we can therefore rewrite (53) as

$$
\frac{k}{2} \int_{0}^{\lambda / 2} B(s)\left[\cot \frac{k}{2}(\xi-s)-\cot \frac{k}{2}(\xi+s)\right] d s .
$$

Using (54), we rewrite equation (13) as

$$
\begin{aligned}
\pi c^{2} F^{\prime \prime}(\xi)=\frac{k}{2}(\mathrm{PV}) \int_{0}^{\lambda / 2} & {\left[\frac{C_{1} F^{\prime}}{F^{3}}+\sigma F^{\prime \prime \prime}-D F^{\prime}\right] } \\
\times & {\left[\cot \frac{k}{2}(\xi-s)-\cot \frac{k}{2}(\xi+s)\right] d s }
\end{aligned}
$$

We determine the coefficients $f_{n}$ and $c^{2}$ by substituting (52) into (55). This is done numerically in the following way. First, we truncate the infinite series in (52) after $M-1$ terms and evaluate the derivatives $F_{1}^{\prime}, F_{1}^{\prime \prime}$ and $F_{1}^{\prime \prime \prime}$ by differentiating the truncated version of (52) term by term. Next, we introduce the mesh points

$$
s_{I}=\frac{\lambda(I-1)}{2(M-1)}, \quad I=1,2, \ldots, M
$$

and

$$
\xi_{I}^{M}=\frac{s_{I}+s_{I+1}}{2}, \quad I=1,2, \ldots, M-1 .
$$


We then satisfy (55) at the mesh points (57). This gives $M-1$ nonlinear equations for the $M$ unknowns $f_{1}, f_{2}, \ldots, f_{M-1}$ and $c^{2}$, to be solved for given values of $C_{1}, D, \sigma, \lambda$ and $A$. The last equation is obtained by fixing the amplitude of the solution, for example by writing

$$
f_{1}=\alpha
$$

where $\alpha$ is prescribed. The resulting system of $M$ nonlinear algebraic equations with $M$ unknowns is solved by Newton's method. For small values of $\alpha$, we use the weakly nonlinear solution as the initial guess. For larger values of $\alpha$, an already computed solution for a smaller value of $\alpha$ is used as the initial guess.

The Cauchy principal value in (55) is evaluated by the trapezoidal rule with summation over the points (56). The symmetry of the quadrature and of the discretization enable us to evaluate the Cauchy principal value as if it were an ordinary integral. The choice of the trapezoidal rule is motivated by the fact that it is spectrally accurate for periodic solutions.

We first use our numerical code to check the asymptotic solution (38). We choose $\sigma=1.5, A=1.0, \lambda=1, C_{1}=1$ and $D=0.7$. It can be checked that (28) then holds. Relation (21) implies $\epsilon=\alpha k$. For various values of $\alpha$, we calculate the quantity

$$
\mu=\frac{\epsilon^{2} b_{2}}{f_{2}},
$$

where $b_{2}$ is defined by (36). We found the values $\mu=0.93413, \mu=0.98336$, $\mu=0.99583, \mu=0.99896$ and $\mu=0.99999$ for $\alpha=0.2, \alpha=0.1, \alpha=0.05$, $\alpha=0.025$ and $\alpha=0.002$ respectively. As expected, $\mu \rightarrow 1$ as $\alpha \rightarrow 0$.

Next we compare the numerical results with the asymptotic results (50) and (51). We set $\sigma=1.5, A=0.2, \lambda=1$ and $C_{1}=1$. Relation (21) implies $\epsilon=\alpha k$. In order to satisfy (41), we choose

$$
D=2 \sigma k^{2}+\frac{C_{1}}{A^{3}} .
$$

We first present results for $\alpha=0.01$. The asymptotic formula (51) gives $c^{\text {asymp }(+)}=5.3875$ and $c^{\text {asymp(-) }}=5.2472$ with the + and - signs in (49), respectively. The corresponding numerical values of $c$ are $c^{\text {num(+) }}=5.3744$ and $c^{\text {num }(-)}=5.2320$. We then repeated these calculations with $\alpha=0.001$. The asymptotic values are then $c^{\text {asymp (+) }}=5.324377$ and $c^{\text {asymp(-) }}=5.310346$; the numerical values are $c^{\text {num(+) }}=5.324238$ and $c^{\text {num(-) }}=5.310206$. As expected, the agreement improves as $\alpha$ is decreased. The numerical values of the first few coefficients of the solution corresponding to $c^{\text {num(+) }}=5.324238$ are $f_{1}=0.001, f_{2}=0.00049986, f_{3}=-0.000003978, f_{4}=-0.0000003143$ and $f_{5}=0.000000005$, in accordance with (50). This constitutes a check on both the asymptotic and the numerical calculations.

So far, our calculations in the situation where (28) does not hold were restricted to the case of $m=2$. Analytical calculations similar to those in $\$ 3.1 .2$ quickly become intractable as $m$ increases. However, numerical solutions can easily be obtained. We present in Figures 4 and 5 typical profiles for $m=4$ and $m=5$. 


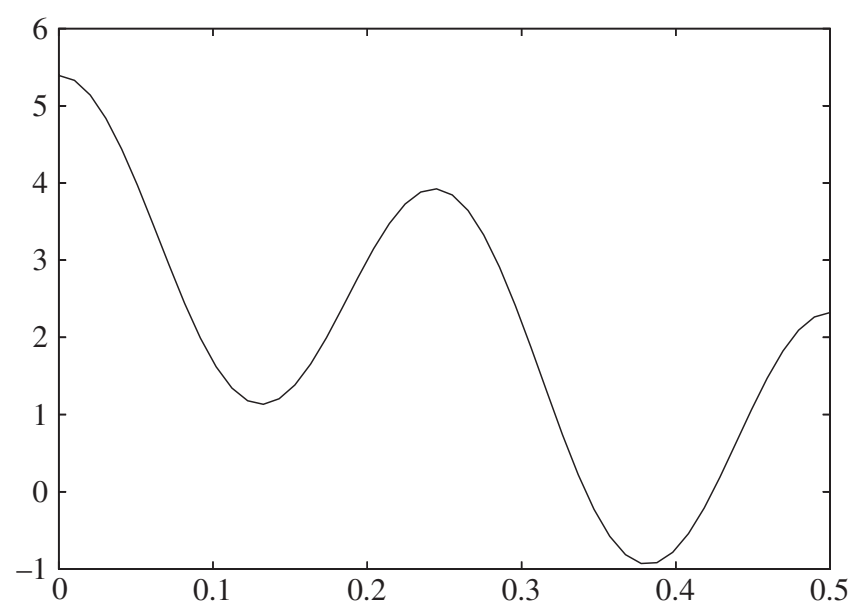

Figure 4: Numerical solution for $m=4$. Only half a wavelength is shown.

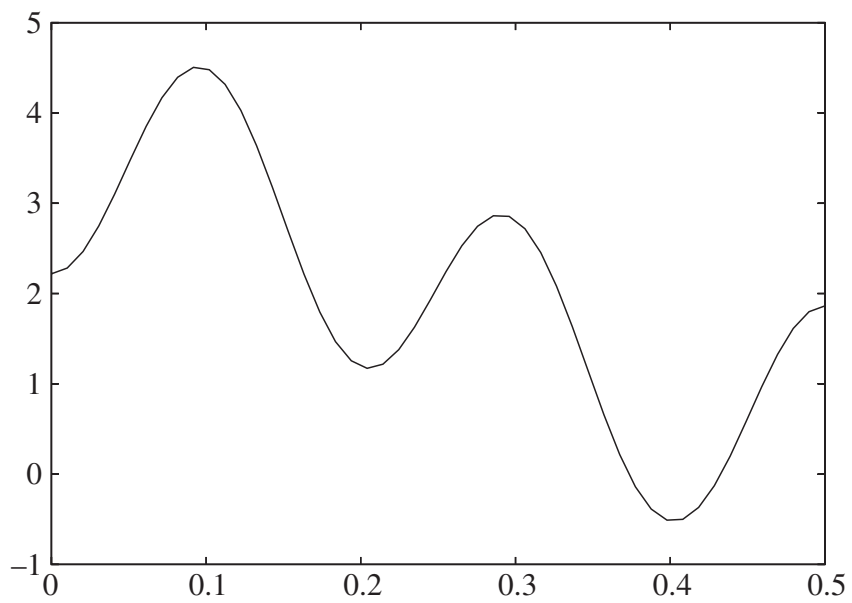

Figure 5: Numerical solution for $m=5$. Only half a wavelength is shown.

§. Final comments. The interactive flow solutions presented in the previous section are believed to be of interest in their own right, and they also influence a wide range of applications, as mentioned in the introduction, as well as raising new questions for investigation. Further work on Wilton ripples, which are typical of interactions between gravity and surface tension, seems warranted for the present setting of near-wall responses. In this context, a study of nonlinear wave evolution that includes full unsteadiness rather than just travelling-wave forms would be of much interest. Analysis of extreme parameter effects, such as those associated with the gravity factor $D$, is also called for.

Numerous extensions appear possible from the model. The present study has focused on relatively thin horizontal layers with the gravity force directed 
towards the underlying solid surface. If the gravity force is in the opposite direction, which corresponds to the fluid layers lying just below a horizontal ceiling, then the sign of the coefficient $D$ is reversed in (7) and in subsequent equations. The full repercussions of this sign change remain to be followed through, including applications to dripping rates. Similarly, an inclined floor or ceiling leads to a constant force term being added to the $X$ momentum balance in (7) and thence to the integrand within (9), which merits further study. The extension to three-dimensional interactions for the sake of increasingly realistic modelling should be of great additional interest.

\section{References}

1. B. Chen and P. G. Saffman, Steady gravity-capillary waves on deep water, Part II: numerical results for finite amplitude. Stud. Appl. Math. 62 (1980), 95-111.

2. R. W. Gent, N. P. Dart and G. T. Cansdale, Aircraft icing. Philos. Trans. R. Soc. A 358 (2000), 2873-2991.

3. D. Gueyffier, J. Li, A. Nadim, R. Scardovelli and S. Zaleski, Volume of fluid interface tracking with smoothed surface stress methods for three-dimensional flows. J. Comput. Phys. 152 (1999), 423-456.

4. S. J. Hogan, Some effects of surface tension on steep water waves, Part 2. J. Fluid Mech. 96 (1980), 417-445.

5. S. D. Howison, J. R. Ockendon and J. M. Oliver, Deep- and shallow-water slamming at small and zero deadrise angles. J. Engrg. Math. 42 (2002), 373-388.

6. S. D. Howison, J. R. Ockendon and S. K. Wilson, Incompressible water entry problems at small deadrise angles. J. Fluid Mech. 222 (1991), 215-230.

7. C. Josserand and S. Zaleski, Droplet splashing on a thin liquid film. Phys. Fluids 15 (2003), $1650-1657$.

8. A. King and E. O. Tuck, Thin liquid layers supported by steady air-flow surface traction. J. Fluid Mech. 251 (1993), 709-718.

9. A. King, E. O. Tuck and J.-M. Vanden-Broeck, Air-blown waves on thin viscous sheets. Phys. Fluids A5 (1993), 973-978.

10. A. A. Korobkin, Shallow water impact problems. J. Engrg. Math. 35 (1999), 233-250.

11. A. A. Korobkin, Asymptotic theory of liquid-solid impact. Philos. Trans. R. Soc. A 355 (1997), 507-522.

12. M. B. Lesser and J. E. Field, The impact of compressible fluids. Annu. Rev. Fluid Mech. 15 (1983), 97-122.

13. J. L. Liow, Splash formation by spherical drops. J. Fluid Mech. 427 (2001), 73-105.

14. R. Purvis and F. T. Smith, Large droplet impact on water layers. AIAA Paper 2004-0414, American Institute of Aeronautics and Astronautics 42nd Aerospace Sciences Meeting, 2004.

15. R. Purvis and F. T. Smith, Air-water interactions near droplet impact. European J. Appl. Math. 15 (2004), 853-871.

16. R. Purvis and F. T. Smith, Droplet impact on water layers: post-impact analysis and computations. Philos. Trans. R. Soc. A $\mathbf{3 6 3}$ (2005), 1209-1221.

17. M. Quero, D. W. Hammond, R. Purvis and F. T. Smith, Analysis of super-cooled water droplet impact on a thin layer and ice growth. AIAA Paper 2006-466, American Institute of Aeronautics and Astronautics 44th Aerospace Sciences Meeting, 2006.

18. L. W. Schwartz and J.-M. Vanden-Broeck, Numerical solutions of the exact equations for capillarygravity waves. J. Fluid Mech. 95 (1979), 119-139.

19. F. T. Smith, L. Li and G.-X. Wu, Air cushioning with a lubrication/inviscid balance. J. Fluid Mech. 482 (2003), 291-318.

20. F. T. Smith and R. Purvis, Air effects on droplet impact. AIAA Paper 2005-5184, American Institute of Aeronautics and Astronautics 4th Theoretical Fluid Mechanics Meeting, 2005.

21. J.-M. Vanden-Broeck, Damped waves generated by a moving pressure distribution. European J. Appl. Math. 12 (2001), 357-366.

22. J.-M. Vanden-Broeck and T. Miloh, The influence of a layer of mud on the train of waves generated by a moving distribution of pressure. J. Engrg. Math. 30 (1996), 378-400.

23. J.-M. Vanden-Broeck and F. T. Smith, Surface tension effects on interaction between two fluids near a wall. Quart. J. Mech. Appl. Math. 61 (2008), 117-128. 
24. S. K. Wilson, A mathematical model for the initial stages of fluid impact in the presence of a cushioning fluid layer. J. Engrg. Math. 25 (1991), 265-285.

25. J. R. Wilton, On ripples. Phil. Mag. 29 (1915), 688-700.

Jean-Marc Vanden-Broeck,

Department of Mathematics,

University College London,

Gower St, London WC1E 6BT,

U.K.

E-mail: broeck@math.ucl.ac.uk
Frank T. Smith,

Department of Mathematics,

University College London,

Gower St, London WC1E 6BT,

U.K.

E-mail: frank@math.ucl.ac.uk 